\title{
Multi-layer endoscopic suturing: a novel method of gastric fistula closure $\nabla$
}

\section{(ㄷ)(1) $\odot$}

Authors

Manol Jovani ${ }^{1}$, Linda Zhang ${ }^{1}$, Yuting Huang ${ }^{1}$, Vivek Kumbhari ${ }^{1,2}$

Institutions

1 Division of Gastroenterology and Hepatology, Johns Hopkins Hospital, Baltimore, Maryland, United States

2 Gastroenterology and Hepatology, Mayo Clinic, Florida, United States

submitted 5.2 .2021

accepted after revision 4.5 .2021

\section{Bibliography}

Endosc Int Open 2021; 09: E1520-E1523

DOI 10.1055/a-1517-4405

ISSN 2364-3722

(C) 2021. The Author(s).

This is an open access article published by Thieme under the terms of the Creative Commons Attribution-NonDerivative-NonCommercial License, permitting copying and reproduction so long as the original work is given appropriate credit. Contents may not be used for commercial purposes, or adapted, remixed, transformed or built upon. (https://creativecommons.org/licenses/by-nc-nd/4.0/)

Georg Thieme Verlag KG, Rüdigerstraße 14,

70469 Stuttgart, Germany

\section{Corresponding author}

Vivek Kumbhari, MD PhD, Associate Professor of Medicine, Director of Bariatric Endoscopy, Johns Hopkins Medicine Division of Gastroenterology and Hepatology, 1800 Orleans Street, Baltimore, MD 21287, USA

Fax: +1-443-683-8335

vkumbhari@gmail.com

\section{ABSTRACT}

Background and study aims Current endoscopic methods of treating gastric fistulas are either too complex or have high rates of recurrence. We aimed to provide a novel endoscopic method for robust fistula closure.

Patients and methods This was a single-center, retrospective study of five patients who underwent multi-layer endoscopic suturing for closing of a chronic fistula ( $>4$ weeks). Devitalization of the fistula tract was achieved with argon plasma coagulation, followed by endoscopic suturing of the fistula. Then, endoscopic suturing of the gastric wall surrounding the fistula was performed, creating an overlay of healthy gastric mucosa around the fistula.

Results Technical success (fistula closure on the day of the procedure) was achieved in all five patients, with no complications. After a median follow up of 5 months (range 2-23 months), there was a $100 \%$ clinical success rate (no fistula recurrence).

Conclusions Our single-operator method of multi-layer endoscopic suturing provides a robust fistula closure with minimal to no risk of recurrence. In light of limitations of current fistula closure methods, further investigations are warranted to better define long-term outcomes with it compared to alternative methods.

\section{Introduction}

Chronic gastrocutaneous fistula (GCFs), defined as fistula present for $>4$ weeks, can occur after removal of long-standing percutaneous endoscopic gastrostomy (PEG) tubes, as well as after other interventions, such as removal of the AspireAssist tube (Aspire Bariatrics, King of Prussia, Pennsylvania, United States), usually placed for weight loss. Chronic GCFs tend to be recalcitrant to standard endoscopic therapy because they are often of a large caliber, but short and fibrotic with high-flow output. Surgery has historically been the mainstay of treatment, but new endoscopic alternatives have emerged. These include tissue destruction of the epithelial lining in the tract (using silver nitrate, argon plasma coagulation [APC] or electrocautery) fol- lowed by tract closure using either through-the-scope (TTS) or over-the-scope clips (OTSC), banding, or plugging the fistula tract with collagen, fibrin or other biomaterials [1-5]. These techniques, however, may have only short-term benefit with fistula recurrence over the long term in up to $35 \%$ of patients $[1,2]$. More recently, several variants of endoscopy-assisted transcutaneous suturing techniques have been described [68]. However, these tend to be complex procedures that require more than one operator.

The OverStitch endoscopic suturing device (Apollo Endosurgery, Austin, Texas, United States) has been predominantly used for bariatric-related endoscopy procedures. To our knowledge, only two case reports have described the use of the OverStitch endoscopic suturing device for chronic GCF closure [9, 
10]. In both cases, a single layer directly on the fistula edges was used for closure. While in these two specific cases technical success was achieved, there was no long-term follow up. Anecdotally, eventual failure is not uncommon with this technique. The reason for this is likely multifactorial. First, the chronic nature of the fistula and/or prior attempted therapy results in a lack of surrounding healthy tissue. Second, as there are only a few bites taken, the tension on each bite is high, which increases the risk of the sutures cutting through the tissue, potentially leading to fistula recurrence.

Considering the limitation of the aforementioned methods, we propose the multi-layer endoscopic suturing technique as a simple, single-operator, robust method to close a GCF. This novel technique addresses many of the challenges of the previously described methods.

\section{Patients and methods}

This was a single-center retrospective study of five patients, four of whom underwent endoscopic suturing for chronic GCF and one for postsurgical gastric leak. The GCF was formed due to the AspireAssist tube in four patients. The AspireAssist tube is a $26 \mathrm{~F}$ silicone percutaneous endoscopic gastrostomy tube, with a gastric segment with five aspiration holes to allow stomach contents to be aspirated easily. It is inserted to promote weight loss and can reside in situ for $>1$ year. All procedures were performed by the same operator (V.K). Procedure time was defined as the time from the introduction of the scope to its withdrawal. Technical success was defined as fistula closure on the day of the procedure, and clinical success was defined as no fistula or leak recurrence at last follow up. Patients were followed up until death or December 31, 2020.

This retrospective study was approved by our Institutional Review Board for Human Research and complied with Health Insurance Portability and Accountability Act (HIPAA) regulations (CR00032640/IRB00116742).

\section{Endoscopic multi-layer suturing procedure}

We performed an endoscopic multi-layer suturing procedure in all patients ( $\downarrow$ Video 1 ). First, a standard esophagogastroduodenoscope (EGD; GIF-Q180, OLYMPUS, Tokyo, Japan) was used to apply APC at 50 watts circumferentially at the gastric margins of the fistula to create a $10-\mathrm{mm}$ dark brown rim to promote scarring ( $\triangleright$ Fig. 1, $>$ Fig. 2). APC also was applied to the fistula tract for internal lining devitalization.

The EGD scope was then removed and exchanged with a therapeutic double channel endoscope (GIF-2TH180, OLYMPUS, Tokyo, Japan) preloaded with the Overstitch Generation 2 device (Apollo Endosurgery, Austin, Texas, United States). We used Prolene 2-0 sutures. The scope was advanced to the fistula, which was usually found on the anterior gastric wall. First, we performed closure of the fistula itself. The tissue helix was used to assist in acquisition of full-thickness bites. A running suture was placed by taking a bite through the gastric wall $1 \mathrm{~cm}$ left of the fistula, and then another bite was taken through the gastric wall $1 \mathrm{~cm}$ to the right of the fistula. This pattern was repeated from distal to proximal until the entire fistula was included

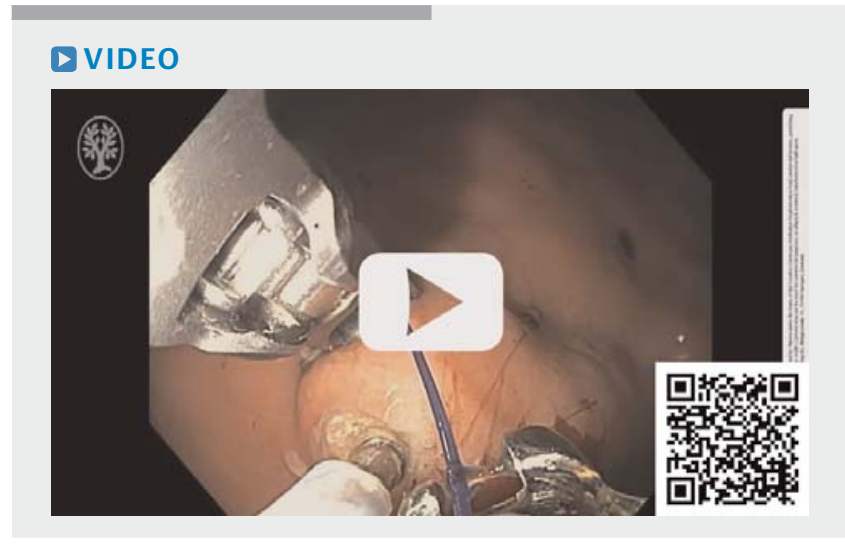

- Video 1 Steps involved in closure of chronic gastrocutaneous fistula with the multi-layer endoscopic suturing technique.

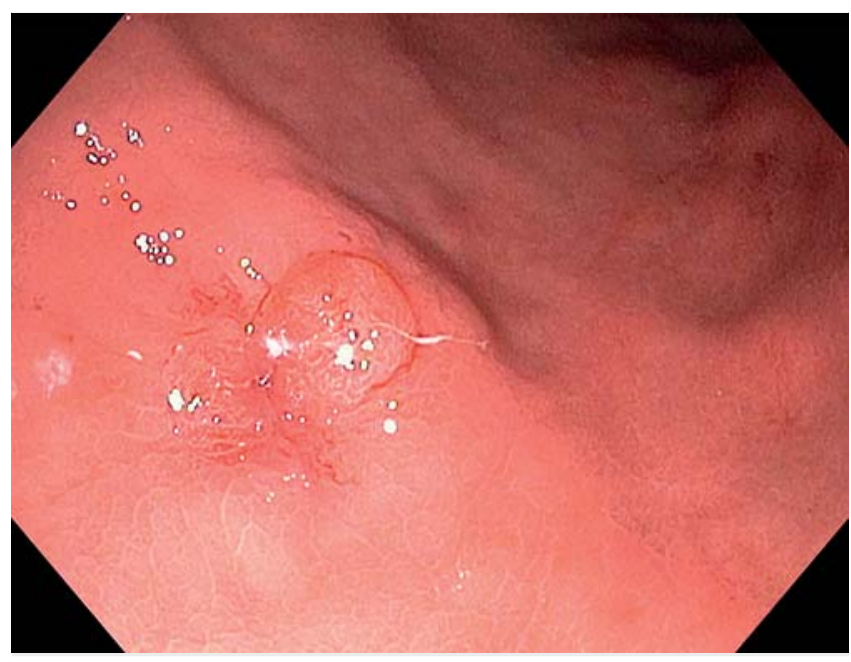

- Fig. 1 Chronic gastrocutaneous fistula prior to treatment.

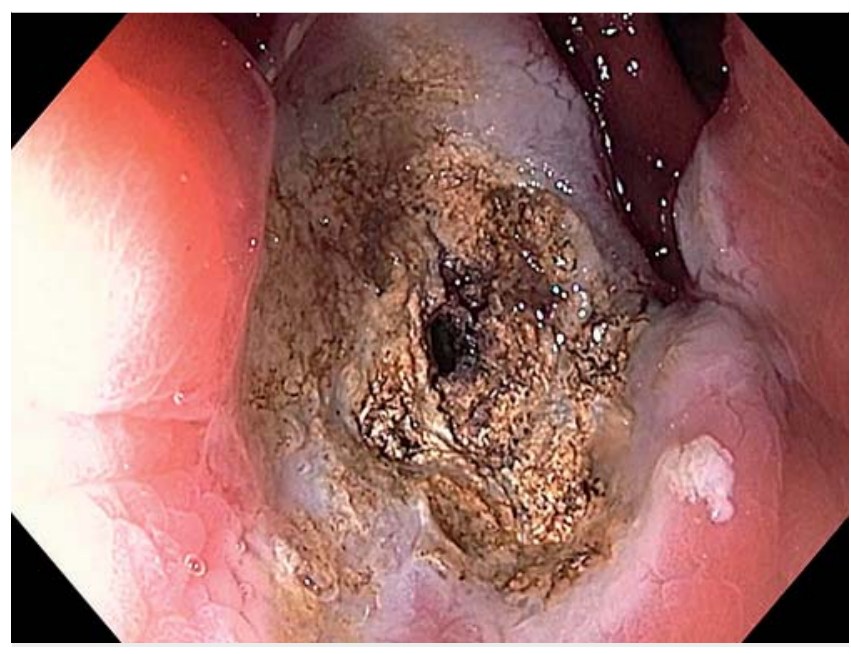

Fig. 2 APC on the gastric margins of the fistula to create a $10-\mathrm{mm}$ dark brown rim to promote scarring. 


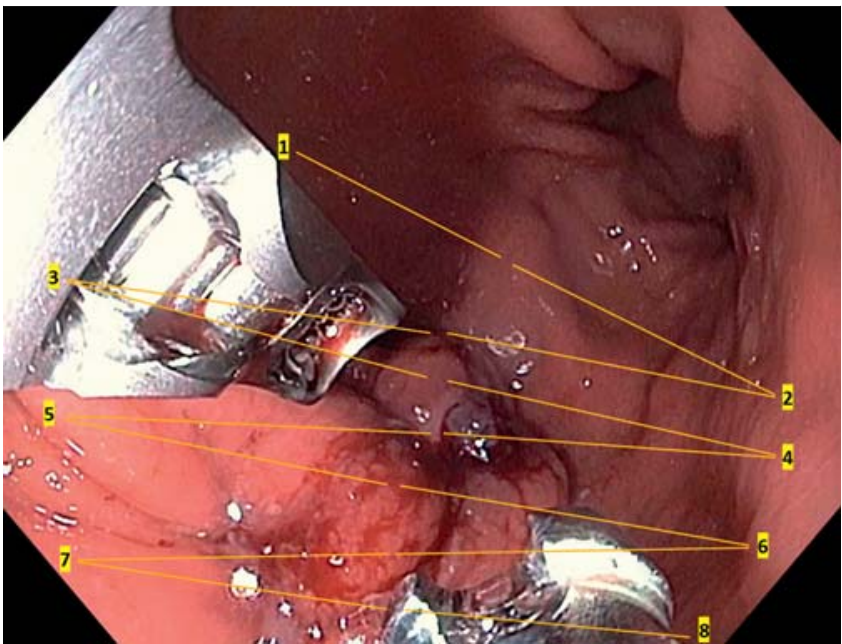

Fig. 3 Fistula closure with endoscopic suturing. Schematic representation of the suturing pattern.

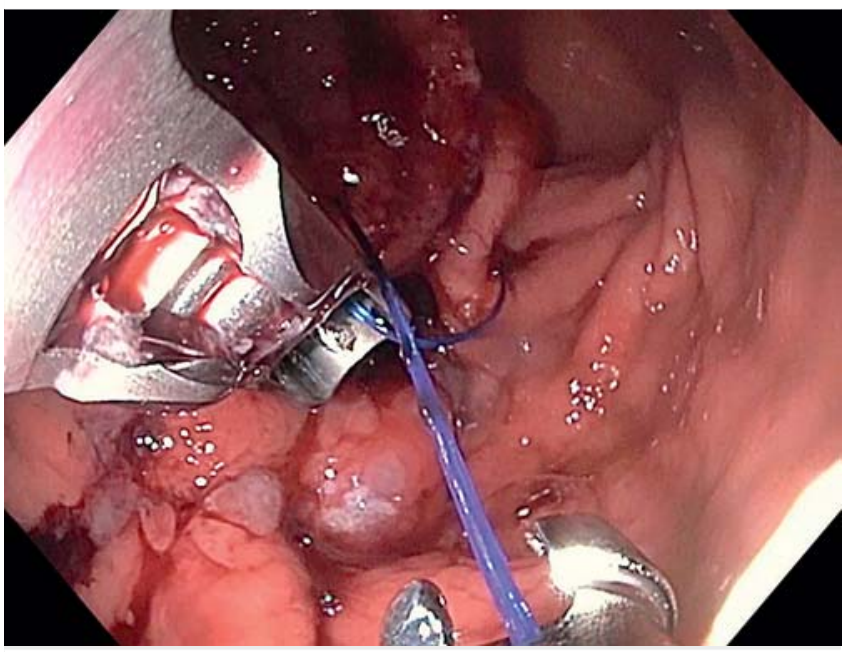

- Fig.4 Initiation of plication of the healthy gastric wall around the fistula site with the purpose of reinforcing its closure.

(usually for 6 bites) Then the T-tag was released and the suture was brought together to form a plication using the cinch, resulting in complete fistula closure ( $>$ Fig. 3 ).

Once the fistula was closed with the first suture, we proceeded to form a second layer on top, with the purpose of reinforcing the GCF closure by plicating the healthy gastric wall around the fistula site. Another suture was loaded into the system, and new full-thickness bites were taken in the healthy gastric wall surrounding the GCF. The first bite was taken distal and to the left of the fistula on the anterior wall, followed by another bite distal and to the right of the fistula on the greater curvature and/or the posterior wall (depending on the exact location of the fistula), followed by a third bite on the anterior wall more proximally and then followed by a fourth bite on the greater curvature and/or the posterior wall, and so on again from distal to proximal ( $\mathbf{F i g . 3 , ~}$ Fig.4). This running suture pattern was repeated for a total of eight bites. Then the T-tag was dropped and the suture was cinched, resulting in closure of the gastric

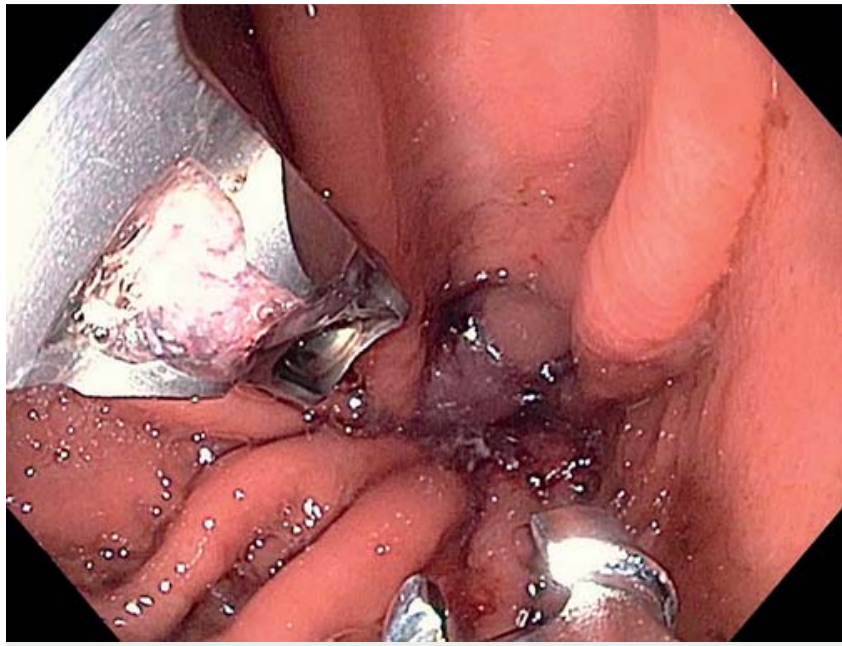

Fig. 5 Successful creation of overlaying of healthy gastric mucosa around the fistula.

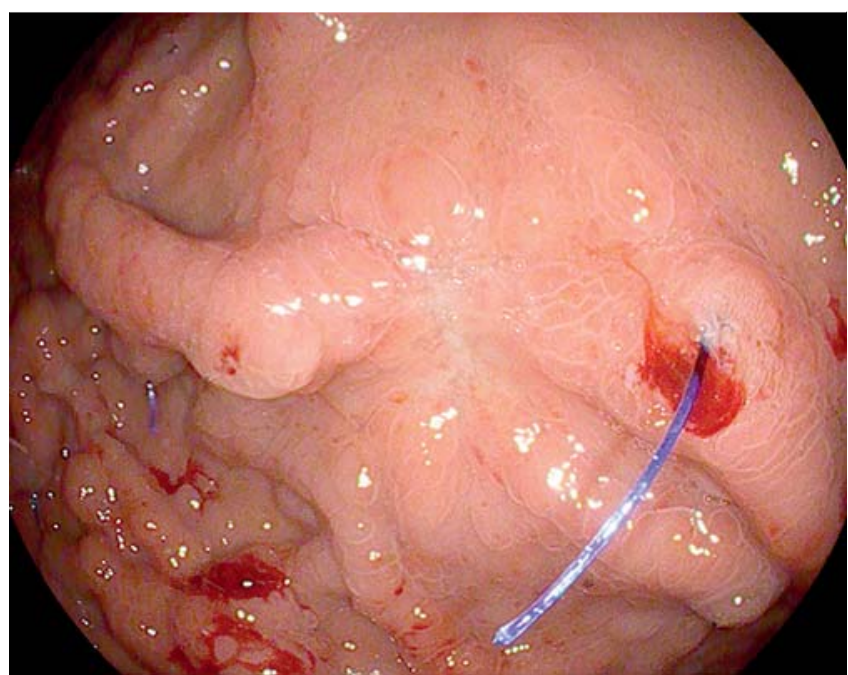

- Fig. 6 View of the fistula about 2 months later, after the sutures were cut to evaluate the fistula site. There was complete healing with a mature scar.

walls over the fistula. If there was sufficient space in the lumen, another suture was loaded into the system taking full-thickness bites in a manner similar to previous, with the aim of achieving a third reinforcing layer. The end result was that the fistula was closed and buried in layers of healthy gastric mucosa ( $\mathbf{F i g . 5}$ ). This resulted in further isolation of the fistula from the gastric content flow, allowing it to close.

\section{Results}

Overall, five patients were included in this study, of whom four (80\%) were women. The average age was 57 years (standard deviation [SD] 14) and the mean BMI was 34.5 (SD 9). All patients were intubated and underwent the procedure under general anesthesia. The mean procedural time was 59 minutes (SD 11). 
Technical success, defined as fistula closure on the day of the procedure, was achieved in all five patients, with no immediate complications as per the American Society for Gastrointestinal Endoscopy lexicon. All patients were discharged home on the same day, with antiemetics and analgesia as needed.

After a median follow up of 5 months (range 2-23 months), no patient died and there was no fistula or leak recurrence (100\% clinical success) ( $\triangleright$ Fig.6). No late adverse events were observed during follow up. We did not have any cases of clinically significant gastric stenosis. As compared to the size of the stomach, we estimate the stomach volume was reduced by about $15 \%$ to $20 \%$ as a result of the procedure, and therefore, we would not anticipate clinically significant weight loss.

\section{Discussion}

Current treatment methods for gastric fistulas are suboptimal in their long-term success, require multiple operators or have complex intra-procedural steps. We describe a novel single-operator method for durable closure of GCFs. We use previously established fistula tract epithelial devitalization, with the addition of endoscopic suturing not only for the fistula itself, but also for multi-layer reinforcement using surrounding healthy gastric mucosa. There are several benefits to this method. First, as opposed to percutaneous suturing methods, it necessitates only one operator and patients do not need to return to remove the transmural stitches. Second, for an operator proficient in endoscopic suturing, this method is an easy addition to clinical practice, because it simply involves additional suturing around the fistula. Third, the multi-layer endoscopic suture may provide a more robust fistula closure than the single-layer endoscopic suture described in two previous case reports $[9,10]$. By increasing the number of bites per suture, the tension on each suture is reduced, thereby decreasing the risk of suture dislodgement by cutting through the gastric wall. In addition, the multi-layer reinforcement further isolates the fistula from gastric contents, enhancing its healing process. In our experience with fistula management, if there are no downstream propagating anastomotic factors (i.e pyloric stenosis) or patient factors (e.g. malnutrition, systemic corticosteroid use) then once a fistula is healed, it is unlikely to recur. While there is a theoretical possibility of weight loss from the reduction of gastric volume, this seems unlikely to us because the reduction in gastric lumen/volume with the purpose of fistula closure is minor. We estimate that the stomach volume was reduced by about $15 \%$ to $20 \%$ as a result of the procedure, and therefore, would not anticipate clinically significant weight loss from it. As a comparison, gastric volume during endoscopic or surgical sleeve gastrectomy is reduced by about $70 \%$.

\section{Conclusions}

In conclusion, we have demonstrated the safe and efficient use of a novel single-operator multi-layer endoscopic suturing procedure for robust and definitive fistula closure. Larger prospective studies are needed to better define its clinical utility and for comparative analysis with currently available endoscopic techniques.

\section{Competing interests}

Dr. Kumbhari is a consultant for Medtronic, Pentax Medical, Boston Scientific, FujiFilm; a consultant for and has received research support from Apollo Endosurgery; and has received research support from ERBE USA.

\section{References}

[1] Currais P, Faias S, Francisco F et al. Gastrocutaneous fistulas after PEG removal in adult cancer patients: frequency and treatment options. Surg Endosc 2020; 35: 2211-2216

[2] Mehfooz A, Muhammad Z, Pophali PA et al. Mo2004 Novel Management strategies for failed PEG related fistulas. Gastrointest Endosc 2016; 83: AB491

[3] Duddempudi S, Ghevariya V, Singh M et al. Treatment of persistently leaking post PEG tube gastrocutaneous fistula in elderly patients with combined electrochemical cautery and endoscopic clip placement. South Med J 2009; 102: 585-588

[4] Singhal S, Changela K, Culliford A et al. Endoscopic closure of persistent gastrocutaneous fistulae, after percutaneous endoscopic gastrostomy (PEG) tube placement, using the over-the-scope-clip system. Therap Adv Gastroenterol 2015; 8: 182-188

[5] Maluf-Filho F, Hondo F, Halwan B et al. Endoscopic treatment of Rouxen-Y gastric bypass-related gastrocutaneous fistulas using a novel biomaterial. Surg Endosc 2009; 23: 1541-1545

[6] Schulman AR, Aihara H, Thompson CC. Treatment of gastrocutaneous fistula after percutaneous gastrostomy placement. Gastrointest Endosc 2016; 84: 851-852

[7] Haito-Chavez Y, Ngamruengphong S, Chen Y-I et al. Novel hybrid technique for closure of refractory gastrocutaneous fistula: endoscopically guided percutaneous suturing. Gastrointest Endosc 2017; 85: 252-253

[8] Moran RA, Brewer Gutierrez O, Yang J et al. Endoscopically guided percutaneous suturing to facilitate closure of a large gastrocutaneous fistula with an over-the-scope clip. Endoscopy 2018; 50: E309-E311

[9] Armengol-Miro JR, Dot J, Abu-Suboh Abadia M et al. New endoscopic suturing device for closure of chronic gastrocutaneous fistula in an immunocompromised patient. Endoscopy 2011; 43: E403-E404

[10] Kantsevoy SV, Thuluvath PJ. Successful closure of a chronic refractory gastrocutaneous fistula with a new endoscopic suturing device (with video). Gastrointest Endosc 2012; 75: 688-690 